\section{Teratoma: a set of teeth in the pelvis}

Teratoma: um conjunto de dentes na pelve

\section{Dear Editor,}

A 25-year-old woman with neither history of trauma nor other previous medical history reported a 2-month history of low back pain. Physical examination revealed no significant abnormality. Conventional abdominal radiography showed the presence of a large, heterogeneous, calcified 10-cm mass in her left lower pelvis (Figures $1 \mathrm{~A}$ and $1 \mathrm{~B}$ ). Pelvic ultrasonography (US) revealed a large heterogeneous mass containing internal hyperechoic areas with acoustic dirty shadowing in the left adnexal area, extending to the rectouterine pouch.

The patient underwent pelvic surgery with left adnexectomy. Macroscopically, the lesion measured $10.3 \times 9.2 \times 8.6 \mathrm{~cm}$ and was filled with a yellowish viscous material, hair, and several tooth fragments (Figure 1C). Analysis of histological specimens confirmed a mature teratoma containing mesodermal, endodermal, and ectodermal tissue.

A series of recent publications in the Brazilian radiological literature have evaluated the role of radiology in the study of abdominal tumors ${ }^{(1-8)}$.

The term "teratoma" comprises several histological types of tumor containing mature or immature tissue of the three germ cell layers: the ectoderm (skin, brain), mesoderm (muscle, fat), and endoderm (mucinous or ciliated epithelium) ${ }^{(9-11)}$. Mature teratoma is the most common benign ovarian tumor in women aged $<45$ years. The clinical manifestations of ovarian teratoma range from an incidentally detected small mass to a malignantly transformed tumor associated with high mortality ${ }^{(10)}$. Most mature cystic teratomas are asymptomatic. Abdominal pain or other nonspecific symptoms occur in a minority of patients ${ }^{(11)}$.

At gross pathological examination, mature cystic teratomas are unilocular and frequently filled with sebaceous material and lined by squamous epithelium. Hair follicles, skin glands, muscle, and other tissues lie within the wall. A raised protuberance (Rokitansky nodule) usually projects into the cyst cavity. At any imaging modality, mature teratomas demonstrate a broad spectrum of findings ranging from purely cystic to mixed masses with components of all three germ cell layers, to noncystic masses composed predominantly of fat. Adipose tissue is present in 67$75 \%$ of cases, and teeth are seen in $31 \%^{(9-11)}$.

Ovarian teratomas may cause various complications (e.g. torsion, rupture, malignant transformation, infection, autoimmune hemolytic anemia) with a wide spectrum of clinical and imaging features ${ }^{(10)}$. At conventional radiography, a typical mature teratoma appears as a large mass with fat opacity and/or multiple toothlike calcifications ${ }^{(9)}$. The most common US finding of an ovarian teratoma is a cystic mass with intratumoral fat and a densely echogenic tubercle (Rokitansky nodule) projecting into the cystic lumen ${ }^{(10)}$. Most mature cystic teratomas can be diagnosed by US, but such diagnosis is complicated by their diversity in appearance ${ }^{(11)}$. The diagnosis of mature cystic teratoma by computed tomography (CT) and magnetic resonance imaging (MRI) is fairly straightforward as such modalities are more fat sensitive. At CT, fat attenuation within a cyst, either with or without calcification in the wall, is diagnostic of mature cystic teratoma. Presence of fat is reported in $93 \%$ of cases and teeth or other calcifications in 56\%. At MRI, the signal intensity of the sebaceous component of a teratoma is similar to that of retroperitoneal fat. Some hemorrhagic lesions may mimic this MRI appearance ${ }^{(11)}$.

\section{REFERENCES}

1. Galvão BVT, Torres LR, Cardia PP, et al. Prevalence of simple liver cysts and hemangiomas in cirrhotic and non-cirrhotic patients submitted to magnetic resonance imaging. Radiol Bras. 2013;46:203-8.

2. Teixeira ACV, Torres US, Westin CEG, et al. Multidetector-row computed tomography in the preoperative diagnosis of intestinal

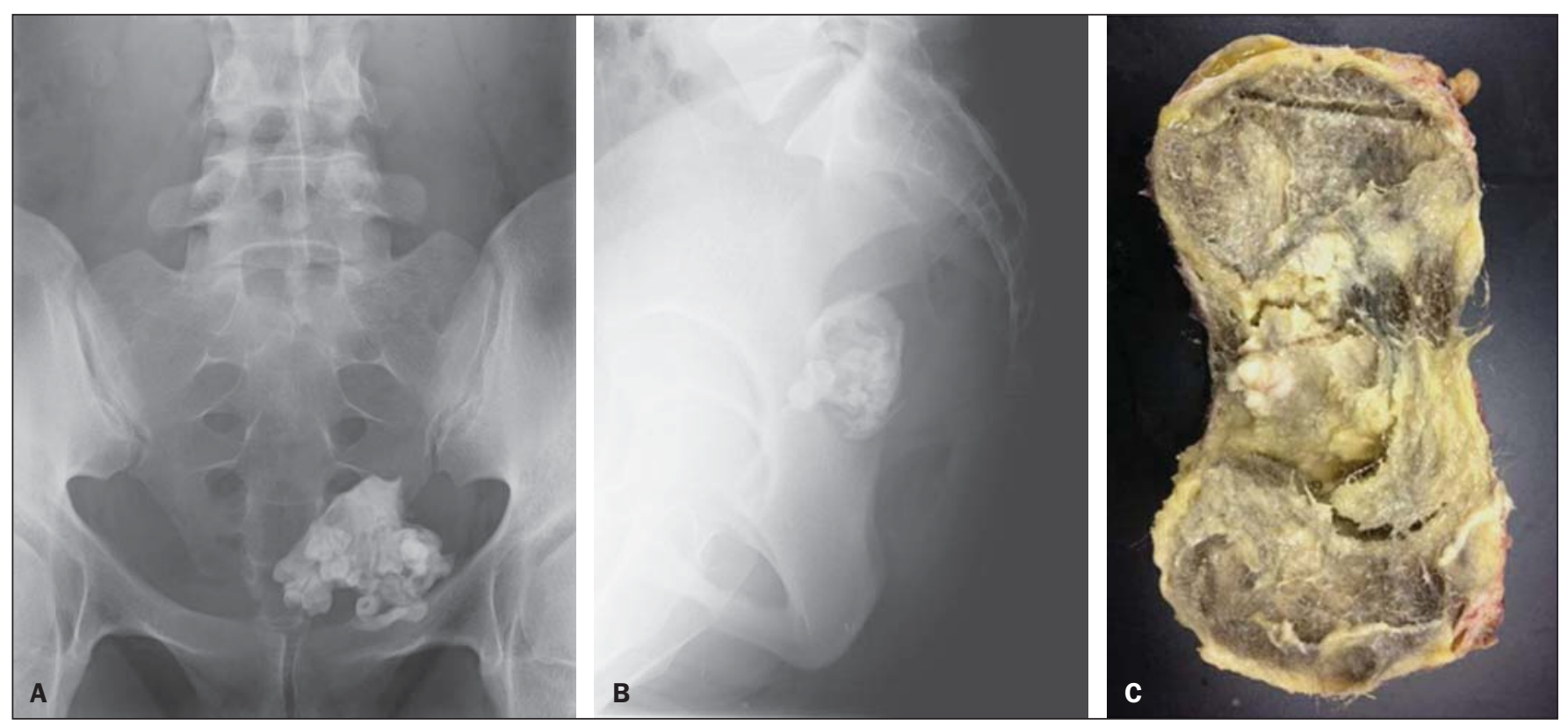

Figure 1. Frontal $(\mathbf{A})$ and lateral $(\mathbf{B})$ radiographic image of the pelvis showing a large calcified mass with multiple toothlike calcifications, indicative of a typical mature teratoma. Photo of the gross specimen $(\mathbf{C})$ showing a well-circumscribed, encapsulated mass measuring $10.3 \times 9.2 \times 8.6 \mathrm{~cm}$. The mass was filled with a yellowish viscous material, hair, and several tooth fragments. 
complications caused by clinically unsuspected ingested dietary foreign bodies: a case series emphasizing the use of volume rendering techniques. Radiol Bras. 2013;46:346-50.

3. Tyng CJ, Bitencourt AGV, Almeida MFA, et al. Computed tomographyguided percutaneous biopsy of pancreatic masses using pneumodissection. Radiol Bras. 2013;46:139-42.

4. Elias Jr J. Imaging findings of unusual hepatic tumors: expanding the differential diagnosis. Radiol Bras. 2014;47(5):ix-x.

5. Silva EJC, Silva GAP. Local behavior and lymph node metastases of Wilms' tumor: accuracy of computed tomography. Radiol Bras. 2014; 47:9-13.

6. Torres LR, Timbó LS, Ribeiro CMF, et al. Multifocal and metastatic hepatic hemangioendothelioma: case report and literature review. Radiol Bras. 2014;47:194-6.

7. Pedrassa BC, Rocha EL, Kierszenbaum ML, et al. Uncommon hepatic tumors: iconographic essay - Part 1. Radiol Bras. 2014;47:310-6.

8. Pedrassa BC, Rocha EL, Kierszenbaum ML, et al. Uncommon hepatic tumors: iconographic essay - Part 2. Radiol Bras. 2014;47:374-9.
9. Jung SE, Lee JM, Rha SE, et al. CT and MR imaging of ovarian tumors with emphasis on differential diagnosis. Radiographics. 2002;22:130525.

10. Park SB, Kim JK, Kim KR, et al. Imaging findings of complications and unusual manifestations of ovarian teratomas. Radiographics. 2008; 28:969-83

11. Outwater EK, Siegelman ES, Hunt JL. Ovarian teratomas: tumor types and imaging characteristics. Radiographics. 2001;21:47590.

Thiago Krieger Bento da Silva ${ }^{1}$, Guilherme Jaquet Ribeiro ${ }^{1}$, Felipe Alba Scortegagna ${ }^{1}$, Gláucia Zanetti ${ }^{2}$, Edson Marchiori ${ }^{2}$

1. Department of Radiology, Hospital São Lucas - Pontifícia Universidade Católica do Rio Grande do Sul (PUCRS), Porto Alegre, RS, Brazil. 2. Department of Radiology, Faculty of Medicine, Universidade Federal do Rio de Janeiro (UFRJ), Rio de Janeiro, RJ, Brazil. Mailing Address: Dr. Edson Marchiori. Rua Thomaz Cameron, 438, Valparaíso. Petrópolis, RJ, Brazil, 25685 120. E-mail: edmarchiori@gmail.com.

http://dx.doi.org/10.1590/0100-3984.2015.0034

\section{Dactilose espontânea (ainhum)}

Dactylolysis spontanea (ainhum)

\section{Sr. Editor,}

Paciente do sexo feminino, 76 anos de idade, branca, com reabsorção óssea nos quintos pododáctilos. Há três anos apresentou intensa dor e edema local. Radiografia convencional (Figura 1) mostrou estreitamento e osteólise das quintas falanges médias e distais, mais acentuados à esquerda, associados a redução focal e concêntrica da espessura das partes moles nas raízes destes dedos. Devido à intensa dor local, a paciente foi submetida a amputação cirúrgica dos quintos dedos dos pés, havendo desaparecimento dos sintomas.

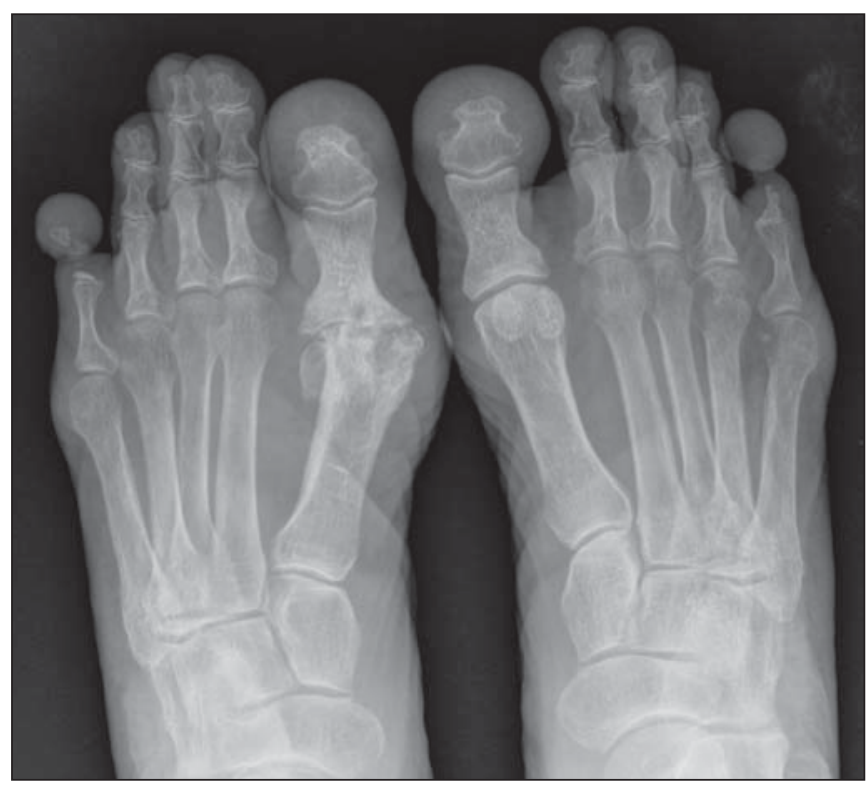

Figura 1. Radiografia dos pés em anteroposterior dois anos após o início dos sintomas. Além do estreitamento e osteólise das quintas falanges médias e distais, sobretudo à esquerda, observam-se alterações degenerativas incidentais na articulação metatarsofalângica do hálux direito.

Várias condições espontaneamente dolorosas nos membros superiores e inferiores, especialmente em suas extremidades, têm sido observadas e relatadas no Brasil ${ }^{(1-5)}$.

Dactilose espontânea (DE), também conhecida como “ainhum”, é uma rara doença que ocorre principalmente em in- divíduos afrodescendentes do sexo masculino (2:1) com idade entre 30 e 50 anos $^{(6)}$. O termo "ainhum", de origem angolana, significa "serrar". O primeiro relato de caso de DE no Brasil foi de um quilombola na Bahia, tendo sido descrito por Silva Lima em $1867^{(7)}$. A prevalência de DE varia de $0,015 \%$ a $2 \%$ da população em alguns países africanos. Sua prevalência no Brasil ainda não foi estudada.

A literatura relata poucos casos de DE em brancos ${ }^{(8)}$. No Brasil, país de população miscigenada, é possível uma incidência maior desta doença em pessoas de pele clara, mas com alguma ascendência africana, nem sempre evidente no fenótipo.

A principal característica da DE é a formação de um anel fibroso constritivo envolvendo a base de um ou mais pododáctilos, condicionando eversão e absorção das estruturas distais, podendo evoluir para amputação espontânea ${ }^{(9)}$. Recentemente, um caso de DE em quirodáctilos foi relatado ${ }^{(9)}$.

As alterações radiográficas são características e podem ser divididas em quatro fases. A primeira é caracterizada pela formação de um sulco profundo com início ao longo do aspecto medial da porção distal da falange proximal, às vezes dando um aspecto de "ampulheta". A segunda fase evolui com aumento do volume distal à banda constritiva, secundário ao linfedema. A terceira fase é caracterizada por absorção óssea progressiva e a quarta, por amputação espontânea, que ocorre em média com quatro a seis anos de evolução ${ }^{(9)}$.

O diagnóstico diferencial deve ser feito com outras condições que podem levar à formação de anéis fibrosos constritivos, como poroceratose de Mibelli, protoporfiria eritropoiética, esclerodermia, psoríase, plica neuropática, hanseníase, sífilis, doença de Raynaud, diabetes mellitus e siringomielia. Há ainda o pseudoainhum factício, causado por torniquetes ${ }^{(9)}$.

Não há tratamento bem estabelecido para a DE. Excisão do sulco seguida por z-plastia pode aliviar a dor e evitar autoamputação nos estágios iniciais ${ }^{(6)}$. Amputação cirúrgica pode ser recomendada para alívio dos sintomas ${ }^{(10)}$.

A DE é uma doença rara e seu diagnóstico é dificultado pela sua baixa prevalência e apresentação clínica variável. A avaliação radiológica permite o diagnóstico precoce, podendo prevenir a autoamputação.

\section{REFERÊNCIAS}

1. Machado BB, Lima CMAO, Junqueira FP, et al. Magnetic resonance imaging in intersection syndrome of the forearm: iconographic essay. Radiol Bras. 2013;46:117-21.

2. Silveira RB, Lopes FAR, Reis ALB, et al. Dysplasia epiphysealis hemi- 